\title{
Recent Advances in Robot-Assisted Surgery: Soft Tissue Contact Identification
}

\author{
Tamás D. Nagy* and Tamás Haidegger*† \\ *Antal Bejczy Center for Intelligent Robotics, Óbuda University, Budapest, Hungary \\ $\dagger$ Austrian Center for Medical Innovation and Technology (ACMIT), Wiener Neustadt, Austria \\ Email: \{tamas.daniel.nagy, tamas.haidegger $\} @$ irob.uni-obuda.hu
}

\begin{abstract}
Robot-Assisted Minimally Invasive Surgery (RAMIS) is becoming standard-of-care in western medicine. RAMIS offers better patient outcome compared to traditional open surgery, however, the surgeons' ability to identify the tissues with the sense of touch is missing from most robotic systems. Regarding haptic feedback, the most promising diagnostic technique is probably palpation; a physical contact examination method through which information can be gathered about the underlying structures by gently pressing with the fingers. In open surgery, palpation is widely used to identify blood vessels, tendons or even tumors; and the knowledge on the exact location of such elements is often crucial with respect to the outcome of the intervention. This paper presents a review of the actual research directions in the field of palpation in RAMIS.
\end{abstract}

Index Terms-Palpation, Surgical Robotics, Haptic Feedback, Robot-Assisted Minimally Invasive Surgery.

\section{INTRODUCTION}

The advancements of the last few decades reshaped the face of surgical interventions radically. The technique of Minimally Invasive Surgery (MIS) started a revolution, when operating through small incisions using so-called laparoscopic instruments, while the visual feedback is provided by endoscopic cameras. This technique offered a number of benefits, such as faster recovery or lower risk of complications, and so became a standard in clinical practice across specialities. MIS also presented new challenges to the surgeons, like the limited range of motion or operating in cumbersome body positions. RobotAssisted Minimally Invasive Surgery (RAMIS) appeared to ease these difficulties; the surgeon is able to operate in a comfortable, seated position at the master console, while their motion is copied by the slave, or patient side instruments. These teleoperated systems - of whom probably the most famous is the da Vinci (Intuitive Surgical Inc., Sunnyvale, CA)—offer enhanced vision and dexterity alongside superior ergonomy [1], [2].

In the case of traditional, open surgical practice, manual palpation is frequently used to gain information about the deeper, non-visible layers of soft tissue. This way the surgeon can identify anatomies with different stiffness to the surroundings, like nerves, blood vessels and tumors as well, since cancerous tissue is usually harder than its environment. In MIS, tissue stiffness is commonly investigated using a procedure called instrument palpation - the tissue is palpated by a long instrument through the trocar, however this technique is less accurate and less sensitive than manual palpation [3]. Unfortunately, most of the current RAMIS systems still lack the ability of force sensing and haptic feedback, thus instrument palpation is infeasible.

\section{Methods}

In this review, we followed the Preferred Reporting Items for Systematic Review and Meta-Analysis (PRISMA) [4]. To find relevant publications in the field of haptics and palpation in RAMIS, the databases PubMed and Google Scholar were used. Since this paper focuses mainly on palpation in RAMIS, the area of haptic feedback is only briefly addressed. During the search procedure the keywords 'palpation', 'surgery', 'stiffness', 'feedback', 'sensor' and 'autonomous' were used together. After the analysis according to PRISMA (Fig. 1.), 35 relevant studies remained from the area of palpation, those are organized into Table I, with the following columns:

- 'Force sensing method': force sensing techniques are divided to thee categories: direct, indirect and visionbased

- 'Platform': the robotic platform the project based on;

- 'Key aspect of the study': brief description of the results;

- 'Form of evaluation': the method used to validate the results;

- 'Year': the year the paper was published;

- 'Ref.': reference to the paper;

The spatial distribution of RAMIS palpation research is overviewed on a map (Fig. 2.), alongside the current da Vinci Research Kit (DVRK) locations [5].

\section{HAPTIC FEEDBACK}

In the human body, at least six types of receptors are reliable for haptic sensation. Basically, all of these are measuring force induced deformations, and can be divided into two groups; tactile and kinaesthetic sensors. Tactile receptors are sensible to higher frequencies, and located in the skin, with varying density all over the body, e.g., the skin on the fingers is quite rich in those. In contrast, kinaesthetic receptors are located mostly in muscles, joints and tendons, and are sensible in a lower dynamic range [6].

Haptic feedback could be useful in a number of manners during RAMIS interventions. However, neither the da Vinci, neither the vast majority of other commercialized RAMIS systems possesses this function. Surgeons might benefit from 


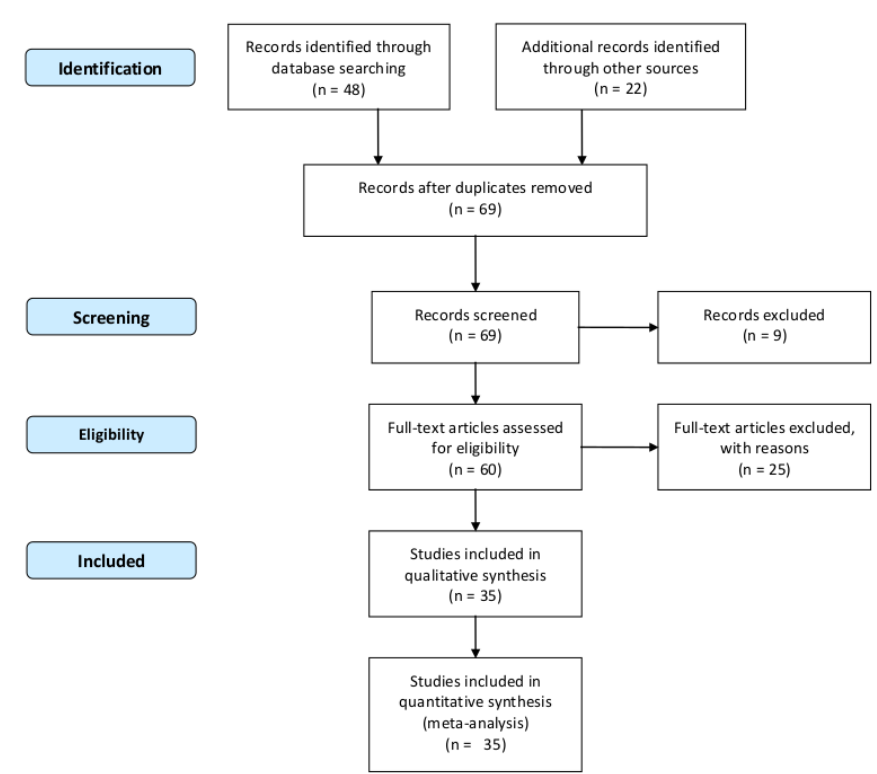

Fig. 1. Flow chart of the selection process according to the Preferred Reporting Items for Systematic Reviews and Metaanalyses (PRISMA) statement.

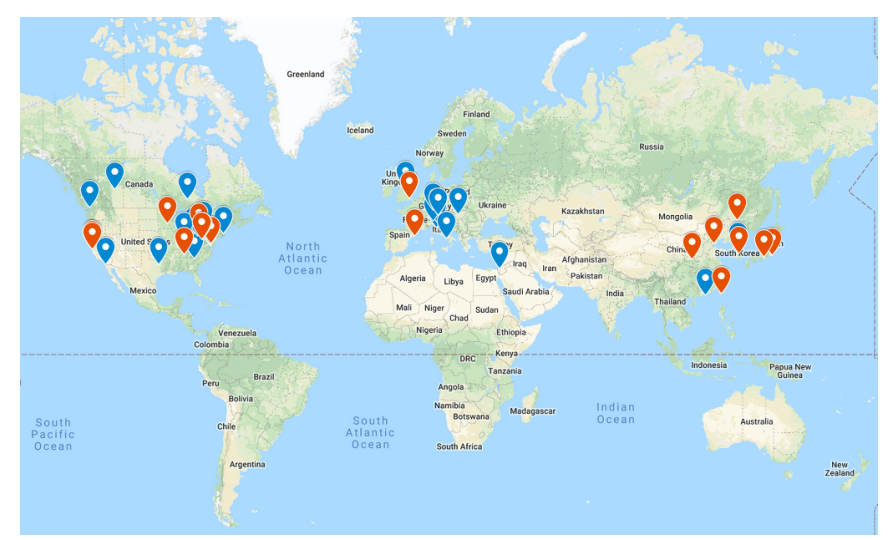

Fig. 2. Geographical distribution of institutes working on robotic palpation worldwide (marked with red pins), compared to the DVRK locations (blue pins).

feeling the tension of the thread during suturing, the gripping force during tissue manipulation, and also collecting information via palpation [7]. The following sections present the different aspects of haptic feedback in RAMIS.

\section{A. Clinical aspects}

In general, safety is a critical aspect of RAMIS, and haptic feedback has the potential to offer improvements in this field. Patient safety could be enhanced e.g., by preventing the surgeon to enter prohibited regions and thus damage sensitive tissues, like blood vessels and nerves, or simply limiting the maximum amount of force the surgeon can apply. Beyond safety, haptics could support the decision making as well, by letting the surgeon know or feel the amount of external or grip force applied. Furthermore, providing palpation and tactile information to the surgeon would also offer diagnostical benefits [8]-[10]. In RAMIS, surgical training is exceptionally important, and haptic feedback has even more advantages for novices [11]. Utilizing haptic feedback during the training (e.g., manipulating in a simulated environment) the trainees can link the applied forces to vision, thus later, working on a system without haptic feedback, they will be able to estimate those forces based on vision solely.

On the patient side, the most basic requirements towards sensorized instruments providing haptic feedback are sterilizability and bio-compatibility. The cleaning of the instruments is commonly performed by autoclave, and most of the available force sensors would not endure such high temperatures. Important to note that the size of the instruments and the trocars is limited as well. Furthermore, due to the clinical usage, the reliability, accuracy and the cost of such instruments are important parameters as well.

\section{B. Technical aspects}

Forces and tactile information can be presented to the surgeon in a number of ways. Probably the most straightforward is to display the used forces on the endoscopic camera screen with different colours; tissue properties or tactile information can also be visualized using augmented reality overlay, or in a separate diagram. Haptic information can be presented to the surgeon through the master controllers by force feedback control method as well [7], [12]-[20]. Moreover, there are a number of methods to display fine tactile information, e.g., air flow, vibration or deformation of the skin [14], [21]-[25]. Displaying the force applied by the jaws of the grippers has also a number of advantages, fastening tissue manipulation, using the minimal force required [26]-[30].

Evidently, all of the different haptic displays require different sensors at the patient side instruments. There is a wide palette of usable force sensing techniques is RAMIS, those are presented in details below, in the palpation section. Moreover, advanced tactile sensorization is currently under intensive research, e.g. using force sensor arrays, based on pressure measurement or optical phenomena [15], [16], [23], [31]-[33].

\section{PALPATION}

Palpation is a medical technique, where the physician examines tissues by the sense of touch, to gain information on the underlying structures. This method is widely used in interventional medicine for diagnostic purposes. In the traditional, open surgical practice, palpation is commonly used for the identification of tissues (e.g., to find damage) find certain organs (e.g., blood vessels) or lesions (e.g., tumors). It is probably the most promising aspect of soft tissue contact identification to be implemented in RAMIS, since technically it is relatively easy to achieve, yet highly beneficial for the surgeon. However, the addition of force sensing capability to RAMIS instruments is problematic due to the limited space and sterilization requirements. 


\begin{tabular}{|c|c|c|c|c|c|c|}
\hline \# & $\begin{array}{c}\text { Force sensing } \\
\text { method }\end{array}$ & Platform & Key aspect of the study & $\begin{array}{l}\text { Form of } \\
\text { evaluation }\end{array}$ & Year & Ref. \\
\hline 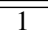 & direct & other & Tumor identification using kinaesthetic feedback & ex vivo & 2008 & [34] \\
\hline 2 & direct & da Vinci & Tissue property estimation and visual overlay in RAMIS & ex vivo & 2009 & [35] \\
\hline 3 & direct & other & Prostate stiffness mapping using tactile sensor & ex vivo & 2011 & [36] \\
\hline 4 & direct & other & Utrasound elastography in prostatectomy & ex-vivo & 2012 & [37] \\
\hline 5 & direct & other & MRI-compatible piezoelectric palpation sensor & in vitro & 2012 & [32] \\
\hline 6 & direct & Phantom Premium & Haptic feedback and augmented reality in RAMIS & in vitro & 2012 & [38] \\
\hline 7 & direct & Phantom Premium & Identification of hard inclusions using machine learning & in vitro & 2013 & [39] \\
\hline 8 & direct & other & Endoscopic stiffness probe for soft tissue identification & in vitro & 2014 & [40] \\
\hline 9 & direct & DVRK & Deflection-based single-use palpation probe & in vitro & 2015 & [41] \\
\hline 10 & direct & da Vinci & Force sensing on the back of EndoWrist instruments & in vitro & 2015 & [42] \\
\hline 11 & direct & DVRK & Autonomous tumor loc. with Gaussian Process adaptive sampl. & in vitro & 2016 & [43] \\
\hline 12 & direct & DVRK & Anutonomous tumor palpation and resection & in vitro & 2016 & [44] \\
\hline 13 & direct & other & Soft robotic skin for autonomous palpation & in vitro & 2017 & [25] \\
\hline 14 & direct & DVRK & Force-controlled exploration to update geom. f deformed env. & in vitro & 2017 & [45] \\
\hline 15 & direct & other & Hard inclusion identification using rolling indentation probe & in vitro & 2017 & [46] \\
\hline 16 & direct & other & Soft robotic probe with stiffness control for palpation & in vitro & 2017 & [47] \\
\hline 17 & direct & Phantom Omni & Prostate cancer localization using rolling indentation probe & ex vivo & 2017 & [48] \\
\hline 18 & direct & da Vinci & Force sensor integrated into da Vinci instrument shaft & in vitro & 2017 & [49] \\
\hline 19 & direct & other & Inexpensive triaxial force sensor for MIS & in vitro & 2017 & [50] \\
\hline 20 & direct & da Vinci & Forceps with triaxial force sensing abilities & sensored validation & 2018 & [51] \\
\hline 21 & direct & DVRK & Autonomous search and augmented reality overlay for tumors & in vitro & 2018 & [52] \\
\hline 22 & indirect & other & Wheeled palpation probe using FBG sensory & ex-vivo & 2008 & [53] \\
\hline 23 & indirect & other & Tactile sensing system based on an expandable balloon & ex vivo & 2010 & [31] \\
\hline 24 & indirect & other & Abnormality identification using rolling indentation probe & in vitro & 2011 & [54] \\
\hline 25 & indirect & da Vinci & FBG force sensor system for RAMIS & sensored validation & 2011 & [55] \\
\hline 26 & indirect & other & Force sensing micro-forceps using FBG sensory & in-vivo & 2012 & [56] \\
\hline 27 & indirect & other & Wireless palpation probe & in vivo & 2014 & [57] \\
\hline 28 & indirect & other & Softness measurement using acoustic sensory & in vitro & 2015 & [58] \\
\hline 29 & indirect & da Vinci & Force sensing from the EndoWrist back end & in vitro & 2015 & [42] \\
\hline 30 & indirect & DVRK & Sensorizing da Vinci instrument shaft using FBG sensors & in vitro & 2016 & [59] \\
\hline 31 & indirect & DVRK & External force estimation from motor currents on DVRK & sensored validation & 2017 & [60] \\
\hline 32 & vision & other & Haptic feedback based on visual cues & in silico & 2015 & [61] \\
\hline 33 & vision & other & Vision based force estimation & ex vivo & 2017 & {$[62]$} \\
\hline 34 & vision & other & Video-tactile pneumatic probe to estimate tissue stiffness & in vitro & 2017 & [33] \\
\hline 35 & vision & da Vinci & Force prediction with visual inf. using machine learning & ex vivo & 2018 & [63] \\
\hline
\end{tabular}

TABLE I. The overview of RAMIS palpation literature. The platforms mentioned: da Vinci (da Vinci Surgical System, Intuitive Surgical Inc., Sunnyvale, CA), DVRK (da Vinci Research Kit [5]), Phantom Premium and Phantom Omni (3D Systems, Rock Hill, South Carolina).

The stiffness mapping of a soft tissue area (Fig. 3) can be organized into two basic groups alongside the utilized sampling methods; discrete and continuous. During discrete mapping, certain points of the tissue are compressed with a palpation probe. This can be performed in a rectangular grid, but methods using adaptive sampling density, based on the local stiffness are also exist [43]. The tissue can also be mapped continuously, without lifting the probe; this requires low friction on the surface or a wheeled palpation probe, and advanced control methods [48], [54].

The pressure required for the palpation of soft tissues varies; it highly depends on the examined tissue, and on the size and shape of the probe tip. Important to note that the absolute pressure-and so the stiffness-values are usually less important, rather the stiffness changes in the local area are significant. Due to this fact, the absolute pressure values are often not calculated, but the stiffness is expressed e.g., simply in the form of applied force.

Similarly, indentation depth is also highly dependent of the examined tissue and evaluation method. In general, given the dimension of a RAMIS palpation probe tip, the applied force is
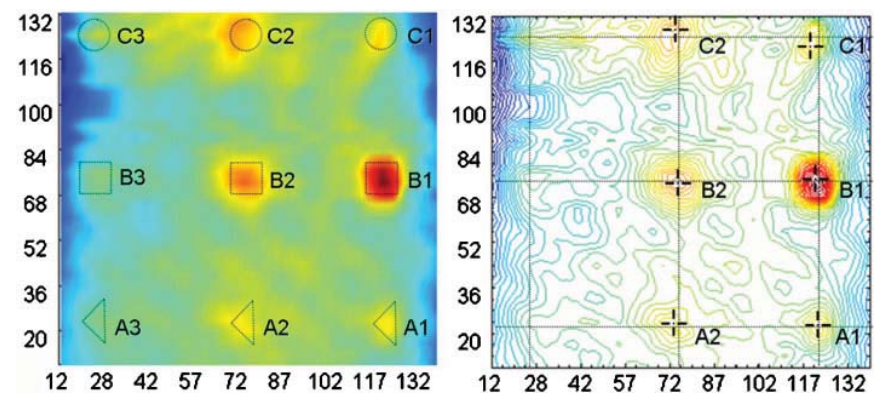

Fig. 3. The stiffness pseudo-colour map (left) and contour map (right) of a silicone phantom with hard inclusions (units: $\mathrm{mm}$ ). The 9 inclusions can be clearly identified on the stiffness map gained by palpation [54].

usually somewhere between $0.1-5 \mathrm{~N}$, and the indentation depth is about $2-8 \mathrm{~mm}$. It is true in general that lower forces are used to examine tissue properties, while higher forces enable structure identification [19], [39], [41], [54].

One of the key aspects of palpation is that how the applied force is measured. In the following sections, different 
implementations on RAMIS palpation are presented, organized alongside the type of utilized sensor modality.

\section{A. Palpation based on direct force sensing}

The most straightforward way to measure the applied force is based on elastic deformation, so called direct force sensing. Common force sensors, like strain gauges or spring-based approaches utilize this principle. The biggest advantage of direct force sensing is the easy implementation and low cost, that enables the development of disposable probes as well. However, the placement of those sensors is problematic, due to the space limitations and requirements of sterilization and cleaning. Some of the force sensors are placed directly on the tooltip of the instrument, along the shaft or even into the driving chain [25], [32], [34]-[52], [59].

One of the first results in RAMIS palpation was presented by Yamamoto et al. [35]. In this study a force sensor was place under the silicone phantom, that was palpated by the da Vinci in teleoperation to identify tissue properties. Another example of the direct approach is presented by a group at UC Berkeley [41]. In this work an inexpensive palpation probe is proposed for the da Vinci. The design is based on the displacement measurement of the spring-attached probe tip (Fig. 4). It was shown that this probe is capable of localizing subcutaneous vessel-like structures of a silicone phantom with high accuracy.

\section{B. Palpation based on indirect force sensing}

Indirect force sensing techniques are also applicable for palpation. The methods do not require electrical components to be inserted into the patient's body, since the applied force is estimated using external sensory. We can find examples in the literature based on acoustic reflection, pressure of the probe's medium or even the motor currents of the robot arms [31], [42], [57], [58], [60]. The drawback of this technique in contrast to the direct force sensing is its complexity and signal processing and computation requirements.

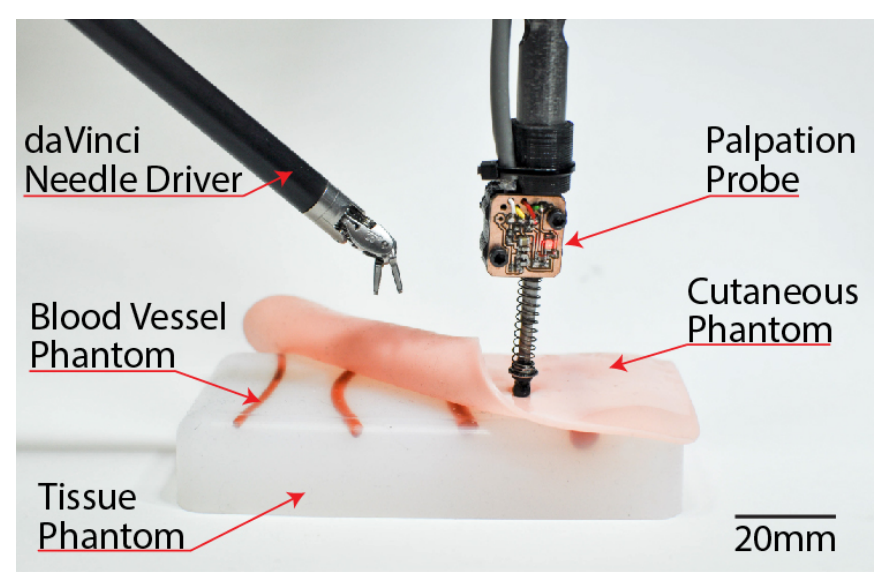

Fig. 4. A disposable palpation probe, mountable on a da Vinci instrument. This spring-based palpation probe was used for locating subcutaneuos blood vessel in phantom environment [41].
In the work of [58], we can find a solution for indirect force sensing. The developed sensor responds to contact position and force at deformable cavity in the sensor probe by using acoustic reflection (Fig. 5).

For RAMIS palpation, probably the most promising force sensors are the Fiber Bragg Grating (FBG) force sensors. FBG sensors are based on the strain-induced shift of Bragg wavelength; a particular wavelength of light that is reflected by the optical fiber, while all other wavelengths are transmitted [64]. These optical fiber sensors possess small physical size and are also tolerate the high temperature during autoclave sterilization, furthermore immune to electromagnetic interference. The mentioned features make FBG force sensors an optimal candidate for RAMIS palpation probes, as already integrated into RAMIS instruments by various research groups (Fig. 6) [53]-[56], [59].

\section{Palpation with vision-based force estimation}

The applied force during RAMIS can also be estimated based on the endoscopic camera images. The major benefit of this technique is that no additional device needs to be added to the operating room setup. Furthermore, bio-compatibility and sterilization are solved already in the case of RAMIS endoscopes. This technique involves several different types of image processing methods, such as feature extraction, filtering of light reflections, and also can be approached using neural networks, but the crucial parts are usually the reconstruction of the tissue surface and handling the inhomogenity in the tissue. Based on the detected deformations of the targeted tissue surface, the applied force values can be estimated [33], [61]-[63], [65]. Despite the mentioned benefits of this technique, the implementation is extremely complex, and usually computationally intense.

One of the most robust solutions for vision-based force sensing was proposed by Aviles et al. [62]. The first step is to recover a 3D deformable structure by extracting the geometry of motion of the hearts surface (Fig. 7). Then, a deep neural network, derives the relationship between the visual-geometric information and the applied force. This solution provided high accuracy results, with an average root-mean square error of $0.02 \mathrm{~N}$.

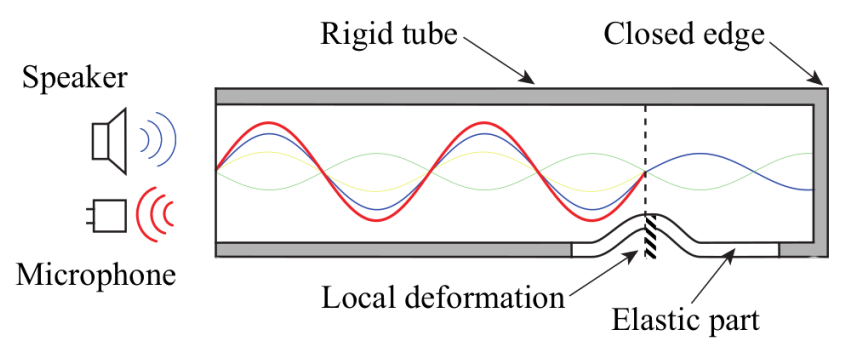

Fig. 5. Acoustic reflection based indirect force sensing. The blue curve represents input wave from speaker, red curve the total wave measured by microphone. The phase delay of the measured wave relates to the magnitude and position of the deformation [58]. 

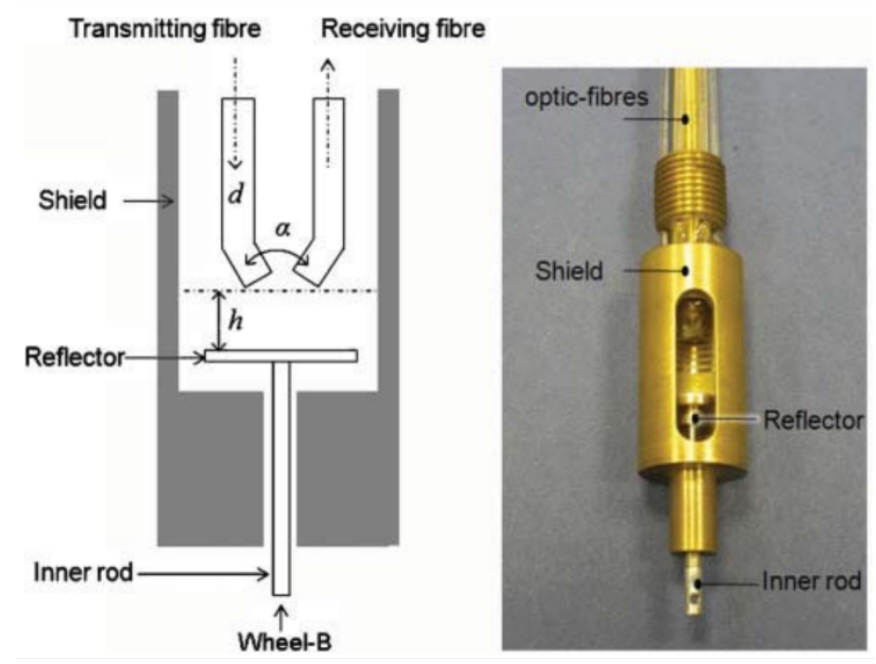

Fig. 6. FBG displacement sensor for RAMIS, developed by Liu et al. [54].
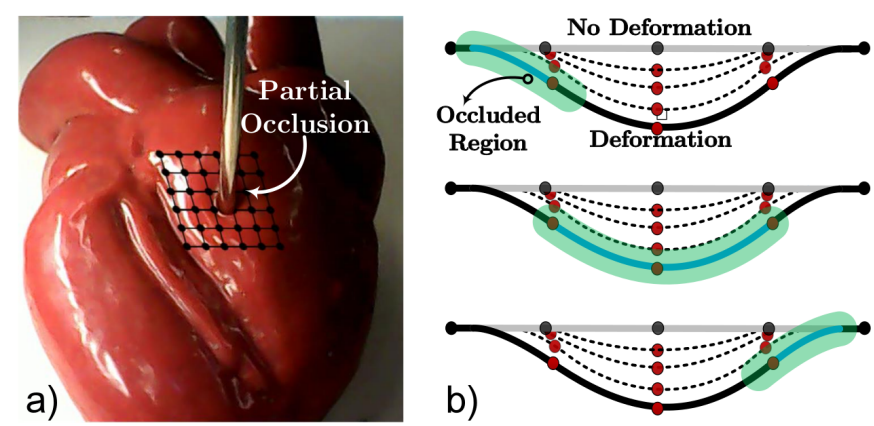

Fig. 7. a) Based on the deformation of the reconstructed surface the applied force can be estimated using deep learning technique. b) The deformation of the occluded regions are recovered from the palpation of other locations [62].

\section{CONCLUSION AND DisCUSSION}

In this study, the current research directions in RAMIS palpation were presented. As of today, there are no commercially available solutions, nevertheless, the last few years showed a significant activity in the field. 35 relevant papers were identified and organized alongside the used sensory methods: 21 utilized direct measurement of the force-induced deformation, 10 measured the contact forces indirectly, via some medium or a driving chain, and in 4 of the processed works the applied force was estimated by enhanced image processing algorithms.

It is generally true that the direct force sensing methods are easier to implement, but are more problematic in terms of required physical space or cleaning abilities. In contrast, indirect and vision-based methods do not rely on electric components inserted into the patient's body, however, generally are more complex. Amongst the mentioned technologies, FBG force sensory promises the biggest chance of breakthrough in this field; FBG-based probes can be small enough physically, more resistant to the high temperature requirements of sterilization, and are also less sensitive to electromagnetic disturbances compared to other force sensors.

\section{ACKNOWLEDGMENT}

This work was partially supported by ACMIT (Austrian Center for Medical Innovation and Technology), which is funded within the scope of the COMET (Competence Centers for Excellent Technologies) program of the Austrian Government. T. D. Nagy and T. Haidegger are supported through the New National Excellence Program of the Ministry of Human Capacities. T. Haidegger is a Bolyai Fellow of the Hungarian Academy of Sciences.

\section{REFERENCES}

[1] A. Takács, D. A. Nagy, I. J. Rudas, and T. Haidegger, "Origins of Surgical Robotics: From Space to the Operating Room," Acta Polytechnica Hungarica, vol. 13, no. 1, pp. 13-30, 2016.

[2] L. Márton, Z. Szántó, T. Haidegger, P. Galambos, and J. Kövecses, "Internet - based Bilateral Teleoperation Using a Revised Time - Domain Passivity Controller," Acta Polytechnica Hungarica, pp. 27-45, 2017.

[3] M. Ottermo, O. Stavdahl, and T. Johansen, "Palpation instrument for augmented minimally invasive surgery," in Proc. of the 2004 IEEE/RSJ International Conference on Intelligent Robots and Systems (IROS), vol. 4. Sendai, Japan: IEEE, 2004, pp. 3960-3964.

[4] D. Moher, A. Liberati, J. Tetzlaff, D. G. Altman, and T. P. Group, "Preferred Reporting Items for Systematic Reviews and Meta-Analyses: The PRISMA Statement," PLOS Medicine, vol. 6, no. 7, Jul. 2009.

[5] P. Kazanzides, Z. Chen, A. Deguet, G. S. Fischer, R. H. Taylor, and S. P. DiMaio, "An open-source research kit for the da Vinci $®$ Surgical System," in Proc. of the IEEE International Conference on Robotics and Automation, Hong Kong, 2014, pp. 6434-6439.

[6] T. A. Kern, "Biological Basics of Haptic Perception," in Engineering Haptic Devices: A Beginner's Guide for Engineers. Berlin, Heidelberg: Springer Berlin Heidelberg, 2009, pp. 35-58.

[7] A. Takács, L. Kovács, I. Rudas, R.-E. Precup, and T. Haidegger, "Models for force control in telesurgical robot systems," Acta Polytechnica Hungarica, vol. 12, pp. 95-114, 2015.

[8] M. Tavakoli, A. Aziminejad, R. V. Patel, and M. Moallem, "Multisensory force/deformation cues for stiffness characterization in softtissue palpation," in Proc. of the Annual International Conference of the IEEE Engineering in Medicine and Biology Society. IEEE Engineering in Medicine and Biology Society. Annual Conference, vol. 1, 2006, pp. 837-840.

[9] M. Moradi Dalvand, B. Shirinzadeh, S. Nahavandi, and J. Smith, "Effects of realistic force feedback in a robotic assisted minimally invasive surgery system," Minimally invasive therapy \& allied technologies: MITAT: official journal of the Society for Minimally Invasive Therapy, vol. 23, no. 3, pp. 127-135, Jun. 2014.

[10] S. Laufer, C. M. Pugh, and B. D. Van Veen, "Modeling Touch and Palpation Using Autoregressive Models," IEEE Transactions on Biomedical Engineering, vol. 65, no. 7, pp. 1585-1594, Jul. 2018.

[11] O. A. J. van der Meijden and M. P. Schijven, "The value of haptic feedback in conventional and robot-assisted minimal invasive surgery and virtual reality training: A current review," Surgical Endoscopy, vol. 23, no. 6, pp. 1180-1190, Jun. 2009.

[12] K. Salisbury and A. Bejczy, "Kinesthetic coupling between operator and remote manipulator," NASA, Technical Report, Jan. 1980.

[13] Y. Kuroda, M. Nakao, T. Kuroda, H. Oyama, and M. Komori, "Interaction model between elastic objects for haptic feedback considering collisions of soft tissue," Computer Methods and Programs in Biomedicine, vol. 80, no. 3, pp. 216-224, Dec. 2005.

[14] Z. F. Quek, W. Provancher, and A. Okamura, "Evaluation of Skin Deformation Tactile Feedback for Teleoperated Surgical Tasks," IEEE Transactions on Haptics, 2018.

[15] M. I. Tiwana, S. J. Redmond, and N. H. Lovell, "A review of tactile sensing technologies with applications in biomedical engineering," Sensors and Actuators, vol. 179, pp. 17-31, Jun. 2012. 
[16] P. S. Girão, P. M. P. Ramos, O. Postolache, and J. Miguel Dias Pereira, "Tactile sensors for robotic applications," Measurement, vol. 46, no. 3, pp. 1257-1271, Apr. 2013.

[17] J. Konstantinova, A. Jiang, K. Althoefer, P. Dasgupta, and T. Nanayakkara, "Implementation of Tactile Sensing for Palpation in Robot-Assisted Minimally Invasive Surgery: A Review," IEEE Sensors Journal, vol. 14, no. 8, pp. 2490-2501, Aug. 2014.

[18] M. Moradi Dalvand, B. Shirinzadeh, A. H. Shamdani, J. Smith, and Y. Zhong, "An actuated force feedback-enabled laparoscopic instrument for robotic-assisted surgery," The international journal of medical robotics and computer assisted surgery: MRCAS, vol. 10, no. 1, pp. 11-21, Mar. 2014.

[19] A. Takács, I. J. Rudas, and T. Haidegger, "Surface deformation and reaction force estimation of liver tissue based on a novel nonlinear mass-spring-damper viscoelastic model," Medical \& Biological Engineering \& Computing, vol. 54, no. 10, pp. 1553-1562, Oct. 2016.

[20] A. Torabi, M. Khadem, K. Zareinia, G. Sutherland, and M. Tavakoli, "Application of a Redundant Haptic Interface in Enhancing Soft-Tissue Stiffness Discrimination," IEEE Robotics and Automation Letters, 2019.

[21] P. Puangmali, K. Althoefer, L. D. Seneviratne, D. Murphy, and P. Dasgupta, "State-of-the-Art in Force and Tactile Sensing for Minimally Invasive Surgery," IEEE Sensors Journal, vol. 8, no. 4, pp. 371-381, Apr. 2008.

[22] M. Bianchi, J. C. Gwilliam, A. Degirmenci, and A. M. Okamura, "Characterization of an air jet haptic lump display," in Proc. of the Annual International Conference of the IEEE Engineering in Medicine and Biology Society, vol. 2011, 2011, pp. 3467-3470.

[23] M. Li, S. Luo, and G. Xu, "A tactile sensing and feedback system for tumor localization," in Proc. of the 13th International Conference on Ubiquitous Robots and Ambient Intelligence (URAI), Xian, China, Aug. 2016, pp. 259-262.

[24] C. Pacchierotti, D. Prattichizzo, and K. J. Kuchenbecker, "Cutaneous Feedback of Fingertip Deformation and Vibration for Palpation in Robotic Surgery," IEEE Transactions on Biomedical Engineering, vol. 63 , no. 2, pp. 278-287, Feb. 2016.

[25] F. Campisano, S. Ozel, A. Ramakrishnan, A. Dwivedi, N. Gkotsis, C. D. Onal, and P. Valdastri, "Towards a soft robotic skin for autonomous tissue palpation," in Proc. of the 2017 IEEE International Conference on Robotics and Automation (ICRA), May 2017, pp. 6150-6155.

[26] N. T. Burkhard, M. R. Cutkosky, and J. R. Steger, "Slip Sensing for Intelligent, Improved Grasping and Retraction in Robot-Assisted Surgery," IEEE Robotics and Automation Letters, vol. 3, no. 4, pp. 41484155, Oct. 2018.

[27] U. Kim, D.-Y. Seok, Y. B. Kim, D.-H. Lee, and H. R. Choi, "Development of a grasping force-feedback user interface for surgical robot system," in Proc. of the 2016 IEEE/RSJ International Conference on Intelligent Robots and Systems (IROS). Daejeon, South Korea: IEEE, Oct. 2016, pp. 845-850.

[28] U. Kim, Y. B. Kim, J. So, D.-Y. Seok, and H. R. Choi, "Sensorized Surgical Forceps for Robotic-Assisted Minimally Invasive Surgery," IEEE Transactions on Industrial Electronics, vol. 65, no. 12, pp. 96049613, Dec. 2018.

[29] C. Lee, Y. H. Park, C. Yoon, S. Noh, C. Lee, Y. Kim, H. C. Kim, H. H. Kim, and S. Kim, "A grip force model for the da Vinci end-effector to predict a compensation force," Medical \& Biological Engineering \& Computing, vol. 53, no. 3, pp. 253-261, Mar. 2015.

[30] J. J. O'Neill, T. K. Stephens, and T. M. Kowalewski, "Evaluation of Torque Measurement Surrogates as Applied to Grip Torque and Jaw Angle Estimation of Robotic Surgical Tools," IEEE Robotics and Automation Letters, vol. 3, no. 4, pp. 3027-3034, Oct. 2018.

[31] Y. Tanaka, Q. Yu, K. Doumoto, A. Sano, Y. Hayashi, M. Fujii, Y. Kajita, M. Mizuno, T. Wakabayashi, and H. Fujimoto, "Development of a realtime tactile sensing system for brain tumor diagnosis," International Journal of Computer Assisted Radiology and Surgery, vol. 5, no. 4, pp. 359-367, Jul. 2010.

[32] A. Hamed, K. Masamune, Z. T. H. Tse, M. Lamperth, and T. Dohi, "Magnetic resonance imaging-compatible tactile sensing device based on a piezoelectric array," Proceedings of the Institution of Mechanical Engineers. Part H, Journal of Engineering in Medicine, vol. 226, no. 7, pp. 565-575, Jul. 2012.

[33] M. M. Gubenko, A. V. Morozov, A. N. Lyubicheva, I. G. Goryacheva, M. Z. Dosaev, M.-S. Ju, C.-H. Yeh, and F.-C. Su, "Video-tactile pneumatic sensor for soft tissue elastic modulus estimation," Biomedical Engineering Online, vol. 16, no. 1, Aug. 2017.
[34] G. L. McCreery, A. L. Trejos, M. D. Naish, R. V. Patel, and R. A. Malthaner, "Feasibility of locating tumours in lung via kinaesthetic feedback," The international journal of medical robotics + computer assisted surgery: MRCAS, vol. 4, no. 1, pp. 58-68, Mar. 2008.

[35] T. Yamamoto, B. Vagvolgyi, K. Balaji, L. L. Whitcomb, and A. M. Okamura, "Tissue property estimation and graphical display for teleoperated robot-assisted surgery," in Proc. of the 2009 IEEE International Conference on Robotics and Automation (ICRA), Kobe, May 2009, pp. 4239-4245.

[36] Q. Peng, S. Omata, D. M. Peehl, and C. E. Constantinou, "Stiffness mapping prostate biopsy samples using a tactile sensor," in Proc. of the Annual International Conference of the IEEE Engineering in Medicine and Biology Society. IEEE Engineering in Medicine and Biology Society. Annual Conference, vol. 2011, 2011, pp. 8515-8518.

[37] I. N. Fleming, C. Kut, K. J. Macura, L.-M. Su, H. Rivaz, C. M. Schneider, U. Hamper, T. Lotan, R. Taylor, G. Hager, and E. Boctor, "Ultrasound elastography as a tool for imaging guidance during prostatectomy: Initial experience," Medical Science Monitor: International Medical Journal of Experimental and Clinical Research, vol. 18, no. 11, pp. 635-642, Nov. 2012.

[38] T. Yamamoto, N. Abolhassani, S. Jung, A. M. Okamura, and T. N. Judkins, "Augmented reality and haptic interfaces for robot-assisted surgery: Augmented reality and haptic interfaces for robot-assisted surgery," The International Journal of Medical Robotics and Computer Assisted Surgery, vol. 8, no. 1, pp. 45-56, Mar. 2012.

[39] K. A. Nichols and A. M. Okamura, "Autonomous robotic palpation: Machine learning techniques to identify hard inclusions in soft tissues," in Proc. of the 2013 IEEE International Conference on Robotics and Automation, Karlsruhe, 2013, pp. 4384-4389.

[40] A. Faragasso, A. Stilli, J. Bimbo, Y. Noh, H. Liu, T. Nanayakkara, P. Dasgupta, H. A. Wurdemann, and K. Althoefer, "Endoscopic addon stiffness probe for real-time soft surface characterisation in MIS," Conference proceedings: ... Annual International Conference of the IEEE Engineering in Medicine and Biology Society. IEEE Engineering in Medicine and Biology Society. Annual Conference, vol. 2014, pp. 6517-6520, 2014.

[41] S. McKinley, A. Garg, S. Sen, R. Kapadia, A. Murali, K. Nichols, S. Lim, S. Patil, P. Abbeel, A. M. Okamura, and K. Goldberg, "A singleuse haptic palpation probe for locating subcutaneous blood vessels in robot-assisted minimally invasive surgery," Aug. 2015, pp. 1151-1158.

[42] T. K. Stephens, Z. C. Meier, R. M. Sweet, and T. M. Kowalewski, "Tissue Identification Through Back End Sensing on da Vinci EndoWrist Surgical Tool 1," Journal of Medical Devices, vol. 9, no. 3, Sep. 2015.

[43] A. Garg, S. Sen, R. Kapadia, Y. Jen, S. McKinley, L. Miller, and K. Goldberg, "Tumor localization using automated palpation with Gaussian Process Adaptive Sampling," in Proc. of the 2016 IEEE International Conference on Automation Science and Engineering (CASE), Fort Worth, 2016, pp. 194-200.

[44] S. McKinley, A. Garg, S. Sen, D. V. Gealy, J. McKinley, Y. Jen, and K. Goldberg, "Autonomous Multilateral Surgical Tumor Resection with Interchangeable Instrument Mounts and Fluid Injection Device,” 2016.

[45] L. Wang, Z. Chen, P. Chalasani, R. M. Yasin, P. Kazanzides, R. H. Taylor, and N. Simaan, "Force-Controlled Exploration for Updating Virtual Fixture Geometry in Model-Mediated Telemanipulation," Journal of Mechanisms and Robotics, vol. 9, no. 2, 2017.

[46] M. Li, J. Konstantinova, G. Xu, B. He, V. Aminzadeh, J. Xie, H. Wurdemann, and K. Althoefer, "Evaluation of stiffness feedback for hard nodule identification on a phantom silicone model," PLOS ONE, vol. 12, no. 3, Mar. 2017.

[47] N. Sornkarn and T. Nanayakkara, "Can a Soft Robotic Probe Use Stiffness Control Like a Human Finger to Improve Efficacy of Haptic Perception?" IEEE Transactions on Haptics, vol. 10, no. 2, pp. 183-195, Apr-Jun 2017.

[48] J. Li, H. Liu, M. Brown, P. Kumar, B. J. Challacombe, A. Chandra, G. Rottenberg, L. D. Seneviratne, K. Althoefer, and P. Dasgupta, "Ex vivo study of prostate cancer localization using rolling mechanical imaging towards minimally invasive surgery," Medical Engineering \& Physics, vol. 43, pp. 112-117, May 2017.

[49] G. A. Fontanelli, L. R. Buonocore, F. Ficuciello, L. Villani, and B. Siciliano, "A novel force sensing integrated into the trocar for minimally invasive robotic surgery," in Proc. of the 2017 IEEE/RSJ International Conference on Intelligent Robots and Systems (IROS), Sep. 2017, pp. 131-136. 
[50] L. Li, B. Yu, C. Yang, P. Vagdargi, R. A. Srivatsan, and H. Choset, "Development of an inexpensive tri-axial force sensor for minimally invasive surgery," in Proc. of the 2017 IEEE/RSJ International Conference on Intelligent Robots and Systems (IROS), Vancouver, BC, Sep. 2017, pp. 906-913.

[51] L. Yu, Y. Yan, X. Yu, and Y. Xia, "Design and Realization of Forceps With 3-D Force Sensing Capability for Robot-Assisted Surgical System," IEEE Sensors Journal, vol. 18, no. 21, pp. 8924-8932, Nov. 2018.

[52] N. Zevallos, A. Srivatsan Rangaprasad, H. Salman, L. Li, J. Qian, S. Saxena, M. Xu, K. Patath, and H. Choset, "A Real-time Augmented Reality Surgical System for Overlaying Stiffness Information," in Proc. of the Robotics: Science and Systems, Jun. 2018.

[53] P. Puangmali, H. Liu, K. Althoefer, and L. D. Seneviratne, "Optical fiber sensor for soft tissue investigation during minimally invasive surgery," in Proc. of the 2008 IEEE International Conference on Robotics and Automation. Pasadena, CA, USA: IEEE, May 2008, pp. 2934-2939.

[54] H. Liu, P. Puangmali, D. Zbyszewski, O. Elhage, P. Dasgupta, J. S. Dai, L. Seneviratne, and K. Althoefer, "An indentation depth-force sensing wheeled probe for abnormality identification during minimally invasive surgery," Proceedings of the Institution of Mechanical Engineers. Part H, Journal of Engineering in Medicine, vol. 224, no. 6, pp. 751-763, 2010.

[55] H. Song, H. Kim, J. Jeong, and J. Lee, "Development of FBG sensor system for force-feedback in minimally invasive robotic surgery," in Proc. of the 2011 Fifth International Conference on Sensing Technology, Nov. 2011, pp. 16-20.

[56] X. He, M. A. Balicki, J. U. Kang, P. L. Gehlbach, J. T. Handa, R. H. Taylor, and I. I. Iordachita, "Force sensing micro-forceps with integrated fiber Bragg grating for vitreoretinal surgery," in Proc. of the Optical Fibers and Sensors for Medical Diagnostics and Treatment Applications XII, vol. 8218. International Society for Optics and Photonics, Jan. 2012.

[57] M. Beccani, C. Di Natali, L. J. Sliker, J. A. Schoen, M. E. Rentschler, and P. Valdastri, "Wireless tissue palpation for intraoperative detection of lumps in the soft tissue," IEEE Transactions on Bio-medical Engineering, vol. 61, no. 2, pp. 353-361, Feb. 2014.

[58] T. Fukuda, Y. Tanaka, M. Fujiwara, and A. Sano, "Softness measurement by forceps-type tactile sensor using acoustic reflection," in Proc. of the 2015 IEEE/RSJ International Conference on Intelligent Robots and Systems (IROS), Hamburg, Germany, Sep. 2015, pp. 3791-3796.

[59] K. S. Shahzada, A. Yurkewich, R. Xu, and R. V. Patel, "Sensorization of a surgical robotic instrument for force sensing," in Proc. of the SPIE BiOS, I. Gannot, Ed., San Francisco, California, United States, Mar. 2016.

[60] H. Sang, J. Yun, R. Monfaredi, E. Wilson, H. Fooladi, and K. Cleary, "External force estimation and implementation in robotically assisted minimally invasive surgery," The International Journal of Medical Robotics and Computer Assisted Surgery, vol. 13, no. 2, Jun. 2017.

[61] M. Li, J. Konstantinova, E. L. Secco, A. Jiang, H. Liu, T. Nanayakkara, L. D. Seneviratne, P. Dasgupta, K. Althoefer, and H. A. Wurdemann, "Using visual cues to enhance haptic feedback for palpation on virtual model of soft tissue," Medical \& Biological Engineering \& Computing, vol. 53, no. 11, pp. 1177-1186, Nov. 2015.

[62] A. I. Aviles, S. M. Alsaleh, J. K. Hahn, and A. Casals, "Towards Retrieving Force Feedback in Robotic-Assisted Surgery: A Supervised NeuroRecurrent-Vision Approach," IEEE Transactions on Haptics, vol. 10, no. 3, pp. 431-443, Jul. 2017.

[63] C. Gao, X. Liu, M. Peven, M. Unberath, and A. Reiter, "Learning to See Forces: Surgical Force Prediction with RGB-Point Cloud Temporal Convolutional Networks," in Proc. of the ISIC 2018: OR 2.0 ContextAware Operating Theaters, Computer Assisted Robotic Endoscopy, Clinical Image-Based Procedures, and Skin Image Analysis, Jul. 2018, pp. $118-127$.

[64] K. Hill and G. Meltz, "Fiber Bragg grating technology fundamentals and overview," Journal of Lightwave Technology, vol. 15, no. 8, pp. 1263-1276, Aug./1997.

[65] A. I. Károly, R. Fullér, and P. Galambos, "Unsupervised Clustering for Deep Learning: A tutorial survey," Acta Polytechnica Hungarica, vol. 15, no. 8, pp. 29-53, 2018. 
T. D. Nagy and T. Haidegger • Recent Advances in Robot-Assisted Surgery: Soft Tissue Contact Identification 